\title{
Multidrug Therapy for Polycystic Kidney Disease: A Review and Perspective
}

\author{
Gianluca Aguiari $^{a} \quad$ Luigi Catizone $^{\mathrm{b}}$ Laura del Senno ${ }^{\mathrm{a}}$

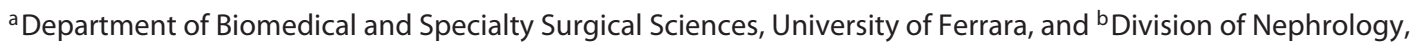 \\ S. Anna Hospital, Ferrara, Italy
}

\section{Key Words}

Autosomal dominant polycystic kidney disease - CAMP, mTOR, and EGFR signaling · Cl-IB-MECA · Therapy

\begin{abstract}
Autosomal dominant polycystic kidney disease (ADPKD) is a renal disorder characterized by the development of cysts in both kidneys leading to end-stage renal disease (ESRD) by the fifth decade of life. Cysts also occur in other organs, and phenotypic alterations also involve the cardiovascular system. Mutations in the PKD1 and PKD2 genes codifying for polycystin-1 (PC1) and polycystin-2 (PC2) are responsible for the 85 and $15 \%$ of ADPKD cases, respectively. PC1 and PC2 defects cause similar symptoms; however, lesions of PKD1 gene are associated with earlier disease onset and faster ESRD progression. The development of kidney cysts requires a somatic 'second hit' to promote focal cyst formation, but also acute renal injury may affect cyst expansion, constituting a 'third hit'. PC1 and PC2 interact forming a complex that regulates calcium homeostasis. Mutations of polycystins induce alteration of $\mathrm{Ca}^{2+}$ levels likely through the elevation of cAMP. Furthermore, $\mathrm{PC} 1$ loss of function also induces activation of $m T O R$ and EGFR signaling. Impaired CAMP, mTOR and EGFR signals lead to activation of a number of processes stimulating both cell proliferation and fluid secretion, contributing to cyst formation and enlargement. Consistently,
\end{abstract}

the inhibition of mTOR, EGFR activity and cAMP accumulation ameliorates renal function in ADPKD animal models, but in ADPKD patients mild results have been shown. Here we briefly review major ADPKD-related pathways, their inhibition and effects on disease progression. Finally, we suggest to reduce abnormal cell proliferation with possible clinical amelioration of ADPKD patients by combined inhibition of CAMP-, EGFR- and mTOR-related pathways.

Copyright $\odot 2013$ S. Karger AG, Basel

\section{Introduction}

Autosomal dominant polycystic kidney disease (ADPKD) is the most frequent hereditary kidney disease accounting for nearly $10 \%$ of dialysis patients with high medical care costs [1]. Cysts occur mostly in the kidney, but also in the liver, ovary, pancreas, spleen and central nervous system. Abnormalities of the vascular system are also reported [1]. Cystogenesis, a focal process involving less than $8-5 \%$ of the nephrons, leads to hypertension in $60 \%$ of ADPKD cases before renal insufficiency and the majority of patients reach ESRD by 55 years of age [1]. Cyst development and enlargement are associated with alterations in epithelial cell growth, fluid secretion and extracellular matrix composition [1]. These processes occur mainly by the impairment of EGFR-, cAMP- and

\section{KARGER}

E-Mail karger@karger.com

www.karger.com/ajn
(C) 2013 S. Karger AG, Basel

0250-8095/13/0372-0175\$38.00/0
Gianluca Aguiari

Department of Biomedical and Specialty Surgical Sciences

University of Ferrara, Via Fossato di Mortara, 74

IT-44121 Ferrara (Italy)

E-Mail dsn@unife.it 


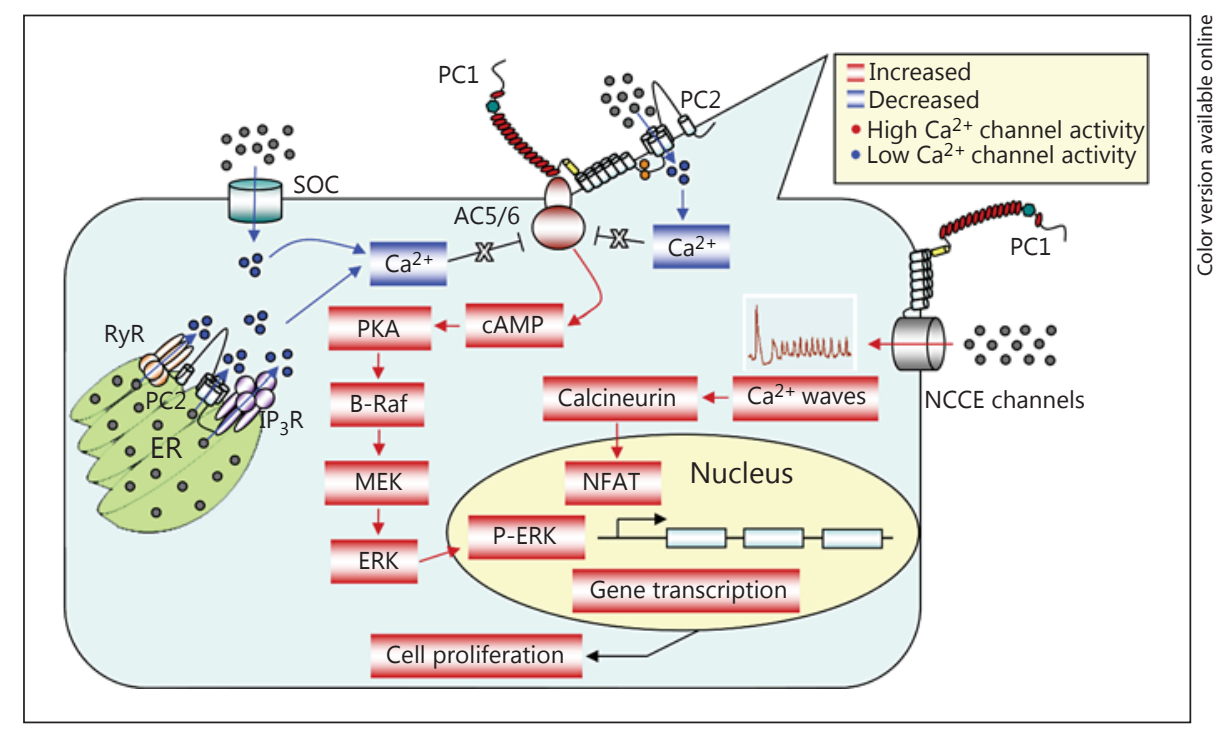

Fig. 1. Abnormal calcium homeostasis and $\mathrm{Ca}^{2+}$-dependent signaling in ADPKD kidney epithelial cystic cell. Polycystin mutations cause abnormal intracellular $\mathrm{Ca}^{2+}$ levels by impairing both plasma membrane and endoplasmic reticulum calcium channels. In particular, in primary cilium, polycystin dysfunction reduces intracellular $\mathrm{Ca}^{2+}$ influx activated by mechanical bending of cilium which protrudes into the tubular lumen. Thus, reduced flow-sensitive intracellular $\mathrm{Ca}^{2+}$ levels and signaling cause a decrease in $\mathrm{Ca}^{2+}$ release from both endoplasmic reticulum (ER) and plasma membrane store-operated channels (SOC) in ADPKD cystic cells. Intracellular $\mathrm{Ca}^{2+}$ reduction may activate $\mathrm{Ca}^{2+}$-dependent adenylyl cyclases 5 and $6(\mathrm{AC} 5 / 6)$ resulting in cAMP accumulation that, by stimulation of Ras/Raf/MEK/ERK signaling, causes an increased cell proliferation. In addition, polycystin loss of function causes an increase in intracellular $\mathrm{Ca}^{2+}$ oscillations through an abnormal activity of plasma membrane NCCE. These $\mathrm{Ca}^{2+}$ oscillations are involved in abnormal cell proliferation of ADPKD cystic cells, by activation of the transcription factor NFAT.
mTOR-mediated pathways [2-4]. In this regard, several drugs have been developed in order to inhibit the signaling associated with ADPKD, including cAMP and $\mathrm{mTOR}$ [5-8]. However, the different molecules, used individually in clinical trials, failed to ameliorate the clinical picture of ADPKD patients.

Here we suggest to treat ADPKD patients with a combination of inhibitors against three major pathways altered in ADPKD such as cAMP, mTOR and EGFR.

\section{Signaling Impaired in ADPKD}

PC1 and PC2 interact forming a complex (PC complex) [9] which works in a number of signaling pathways mainly related to calcium, cAMP, mTOR and EGFR which are involved in the control of cell growth, cell adhesion and apoptosis.

\section{Calcium-Related Signaling}

In primary cilia of kidney epithelium the PC complex functions as a mechanotransductor which regulates in- tracellular calcium influx in response to fluid flow variations (fig. 1). Alterations of $\mathrm{PC} 1$ or $\mathrm{PC} 2$ proteins induce a strong reduction in calcium influx, with aberrant calcium-dependent signals, resulting in altered ciliary function, planar cell polarity and organ morphogenesis [10, 11]. The loss of local calcium affects cAMP homeostasis by de-repressing the $\mathrm{Ca}^{2+}$-sensitive adenylyl cyclases AC5 and AC6, leading to a cAMP increase and the development of renal cysts [12]. Moreover, in PKD1-mutated kidney cystic cells the increase in calcium oscillations, dependent on non-capacitative calcium entry (NCCE) channels (fig. 1), is associated with an increased cell proliferation, via activation of the transcription factor NF-AT [13].

\section{cAMP-Related Signaling}

In normal kidney epithelial cells cAMP elevation has an inhibitory effect on cell growth, while in ADPKD cysts it stimulates cell proliferation. This occurs by activating ERK signaling through the sequential phosphorylation of PKA, B-Raf and MAPK kinases [14] (fig. 1, 2). Moreover, cAMP may also stimulate fluid secretion and cyst en- 


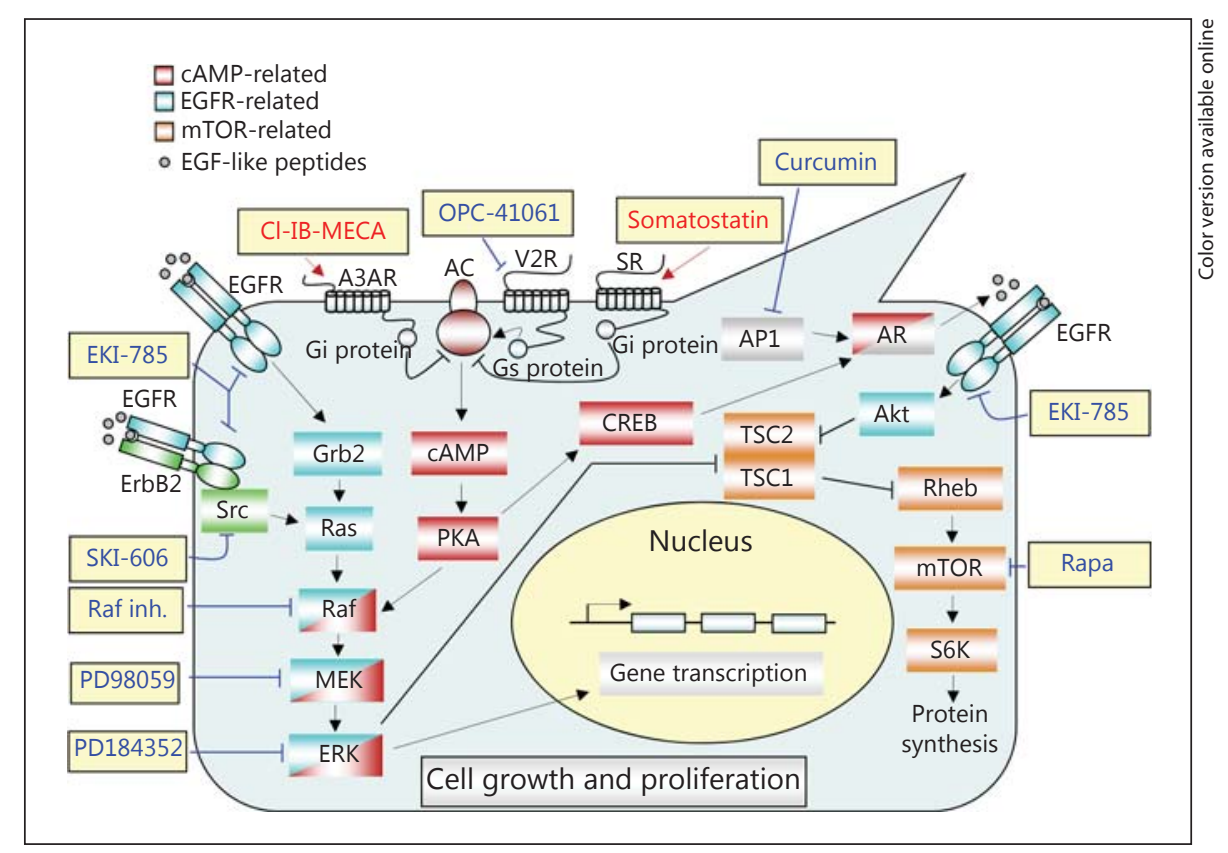

Fig. 2. Altered cAMP-, EGFR- and mTOR-dependent signaling pathways in ADPKD cystic cell and related inhibitors. Polycystin loss of function causes an increase in cell proliferation by alteration of different pathways mostly including cAMP, EGFR and mTOR signaling. The activation of EGFR, which was found to be translocated to the apical membrane in cystic cells, by interaction with its ligands, increases the activity of the Ras/Raf/ERK signaling pathway which stimulates cell growth. EGFR activation is also enhanced by AR which is overexpressed in cystic cells through a CREB- and AP1-

largement, activating the apical CFTR channel and specific basolateral transporters $[14,15]$. Consistently, adenylyl cyclase agonists, beside promoting cell proliferation, are known to be able to stimulate electrolyte secretion in human ADPKD cells in vitro [3].

\section{mTOR-Related Signaling}

ADPKD patients carrying deletions of PKD1 and TSC2 adjacent genes show an earliest cystogenesis, therefore we can assume that both PC1 and tuberin (the TSC2 gene product) function in a common cystogenic pathway. TSC2 is known to inactivate the signaling of the Ser/Thr kinase mTOR (fig. 2) which, in fact, is found abnormally activated in ADPKD cyst-lining cells $[4,16]$. mTOR activation results in increased protein translation via phosphorylation of S6K and 4EBP1 leading to cell growth stimulation and proliferation [4]. PC1 inhibits mTOR activity by interacting with tuberin, thus blocking its inactivation which usually occurs through phosphorylation by ERK and Akt kinases [17-19]. dependent signaling. Abnormal cAMP accumulation also contributes to the activation of the Ras/Raf/ERK signal. Furthermore, this pathway may be stimulated also by activation of Src, which is able to interact with EGFR in its EGFR/ErbB2 heterodimer form [53]. In addition, altered EGFR and CAMP signaling stimulate mTOR activity by activation of Akt and ERK kinases that inhibit the TSC1/TSC2 complex. Inhibitors (blue) and agonists (red) are indicated. Colors refer to the online version only.

\section{EGFR-Related Signaling}

ADPKD cell proliferation may be stimulated also by EGFR through a mechanism involving the sequential activation of Ras, Raf-1, MEK and ERK signaling [14], where the EGFR signal may converge on the same pathway activated by cAMP, which leads to activation of ERK kinases (fig. 2). Consistently, cyst-derived epithelial cells are susceptible to proliferative stimuli of EGF-related peptides which are secreted into the apical medium of cultured ADPKD epithelia as well as in cyst fluids of ADPKD patients [20].

In this regard, the increased expression of TGF- $\alpha$ is also observed in ADPKD cells, and transgenic mice overexpressing TGF- $\alpha$ develop cystic kidneys [21]. Moreover, EGF-related peptides amphiregulin (AR) and heparin-binding EGF are also increased in cystic cells of PKD patients [2]. In particular, AR expression is associated with activation of transcription factors CREB, a cAMP downstream effector, and AP1 (fig. 2) [22]. Furthermore, in ADPKD cystic cells, the apical plasma 
Table 1. Molecular inhibitors with the relative targets against cAMP-, mTOR- and EGFR-dependent signaling pathways

\begin{tabular}{|c|c|c|c|c|c|c|}
\hline Sirolimus & mTOR & yes & yes & yes & failed clinical trials & $7,35,37,48$ \\
\hline Everolimus & mTOR & yes & yes & yes & failed clinical trials & $8,36,38$ \\
\hline Tolvaptan & V2R & yes & yes & yes & in progress clinical trials & 5,26 \\
\hline Octreotide & SR & yes & yes & yes & in progress clinical trials & $6,27-29$ \\
\hline MEK inhibitors & MEK & yes & yes & no & good in PKD cell models, but failed in PKD animals & 32,33 \\
\hline PD184352 & ERK & yes & yes & no & good in PKD cell and animal models & 34 \\
\hline Cl-IB-MECA & A3AR & yes & no & no & good in PKD cell models & 30 \\
\hline Curcumin & AP1 & yes & yes & no & good in PKD cell and animal models & $22,40,41$ \\
\hline EGFR inhibitors & EGFR & yes & yes & no & good in PKD cell and animal models & 24,39 \\
\hline
\end{tabular}

The therapeutic agents currently used in clinical trials for the treatment of ADPKD patients are also indicated.

membrane mislocalization of EGFR, instead of its normal basal localization, as well as the heterodimerization of EGFR with ErbB2 (fig. 2), play a further role in cyst enlargement $[23,24]$. Therefore, the EGFR activation may generate an autocrine loop resulting in increased cell proliferation and cyst expansion in ADPKD cystic cells [23].

\section{ADPKD Treatment}

Currently, different molecules able to reduce cell proliferation and renal cystic volume in ADPKD animal models are being used for clinical trials in ADPKD patients. For instance, inhibitors of mTOR, such as rapamycin (sirolimus) or its derivate everolimus, are already used in clinical trials, while other compounds like cAMP and EGFR inhibitors are still in study on animal models or have to complete clinical trial phases. Here, we describe the effects of some drugs developed for ADPKD treatment (see also fig. 2 and table 1).

\section{cAMP Signal Inhibitors}

In preclinical studies the V2R antagonist OPC-31260 reduces cyclic AMP levels, renal cystogenesis, kidney enlargement and renal dysfunction in the $P \mathrm{kd}^{-/ \mathrm{tm} 1 \text { Som }} \mathrm{Pkd} 2$ mouse model [25]. Consistently, another V2R antagonist, OPC-41061 (tolvaptan), with a high affinity and strength for the receptor, inhibits ERK pathway, cAMP production, $\mathrm{Cl}^{-}$secretion and the in vitro cyst growth of threedimensionally cultured AVP-induced ADPKD cells [26].
These findings encouraged the setting of clinical trials in ADPKD patients using V2R inhibitors. In fact, 3 years of treatment with tolvaptan slows the increase in total kidney volume and kidney decline function in ADPKD patients compared with placebo. However, different side effects as thirst, polyuria, and related adverse events in some patients were observed [5].

Also the octreotide, an analogue of somatostatin that inhibits cAMP accumulation (see fig. 2), has been evaluated for its potential inhibition of cyst growth in ADPKD animal models. Octreotide treatment results to reduce cAMP accumulation and the progression of liver and kidney cysts in a PKD rat model [27]. In a randomized, double-blind, placebo-controlled clinical trial with 42 ADPKD patients, octreotide treatment after the first year maintains TKV almost unchanged, while it increases in the placebo group. Similar benefits are also observed in the second year of treatment, suggesting that octreotide administration may reduce kidney cyst growth $[6,28,29]$.

Furthermore, cAMP levels may be also reduced by the stimulation of adenosine type 3 receptors (A3AR) which inhibits adenylyl cyclase and are overexpressed in ADPKD cystic cells and tissues [30]. The specific A3AR agonist Cl-IB-MECA is able to reduce cell proliferation by reduction of cAMP levels and ERK activity in ADPKD cystic cells [30]. Future studies on ADPKD animal models are necessary to establish if Cl-IB-MECA is effective also in vivo.

Since cAMP elevation activates B-Raf/MEK/ERK pathway in ADPKD cells (fig. 1,2), its inhibition should reduce renal cyst expansion. Accordingly, the treatment 
with sorafenib, a Raf inhibitor, reduces proliferation of cystic cells from human ADPKD kidneys by reducing ERK, B-Raf and MEK/ERK activation. Sorafenib may thus be used as therapeutic molecule to reduce cyst expansion [31].

Although the MEK pathway appears to be crucial in cAMP- and EGFR-dependent cyst formation, the efficacy of MEK inhibitors in PKD cell and animal models results controversial. In fact, the treatment with the MEK inhibitor PD98059 completely inhibits ADPKD cell proliferation in response to cAMP agonists [32]; however, the MEK inhibitor U0126 does not retard the cyst development in a PKD mouse model [33]. Although U0126 does not reduce cyst progression, the treatment with the ERK inhibitor PD184352 slows cyst growth in polycystic kidney disease PKD mice [34]. These contradictory findings indicate that further studies in other PKD animal models are needed to evaluate the efficacy of these molecules.

\section{mTOR Inhibitors}

Since in ADPKD kidneys mTOR kinase activity is increased [4], mTOR inhibition should delay cystic growth and expansion in ADPKD kidneys. Actually, treatments with mTOR inhibitors rapamycin/sirolimus or everolimus decrease renal cyst size and improve kidney function in ADPKD animal models $[35,36]$. Results of a randomized clinical trial suggest that rapamycin administration in ADPKD patients does not modify the GFR compared with standard care-treated group [7]. Moreover, the TKV does not change in sirolimus-treated patients and controls, while urinary albumin excretion rate is higher in the sirolimus-treated group. Thus, 18 months of sirolimus treatment does not improve renal function in ADPKD patients with early chronic kidney disease [7]. Other studies show that after 6 months of treatment with higher doses of sirolimus cyst volume does not change, while it increases in conventional therapy-treated subjects [37]. On the contrary, parenchymal volume increases by sirolimus administration while it is stable with standard therapy. Thus, high doses of sirolimus inhibit cyst growth and increases parenchymal volume in patients with ADPKD after a short period of monitoring. Further studies are needed to understand whether these effects may ameliorate renal function and the quality of life in ADPKD patients [37].

Clinical trials have also been assessed by administration of everolimus which are used in a 2-year study that includes placebo controls and ADPKD patients at a late stage [8]. In everolimus-treated patients, both TKV cyst volume and renal parenchymal volume increase more slowly than in those treated with placebo. However, after 24 months the estimated GFR does not change in the everolimus group. Findings of a 2 -year study suggest that everolimus slows the increase in TKV, but does not improve renal function of ADPKD patients [8]. This discouraging result may be due to late stage of disease, therefore clinical trials should be conducted on patients with early stage of chronic kidney disease, so that everolimus therapy may actually improve the renal function [38].

\section{EGFR Inhibitors}

Administration of EGFR inhibitors EKI-785 and EKB569 in a rat model for ADPKD lowers kidney weights and cyst volumes, suggesting a therapeutic potential of EGFR inhibition for ADPKD treatment [39]. Also the inhibition of EGF-like growth factors such as TGF- $\alpha$, AR, and heparin-binding EGF, found abnormally expressed in human ADPKD epithelial cells $[2,22]$, may be taken in account for ADPKD treatment. In fact, the inhibition of AR by specific antibodies restores normal cell growth in human and mouse ADPKD cystic cells [22]. In addition, the inhibition of both CREB and AP1 activity, which stimulates AR expression in these cells, also reduces cystic cell growth [22]. Consistently, curcumin, a potential AP1 inhibitor that is known to reduce activities of several signaling proteins including ERK and fos, significantly reduces cyst development in an in vitro ADPKD model [40]. Moreover, in Pkd1 gene conditionally inactivated mice, curcumin induces a slower progression of proliferation index, cystic index and kidney growth [41]. The inhibition of Src, a kinase linked to EGFR signaling, by the SKI606 molecule (bosutinib) also reduces cell proliferation in human ADPKD cystic cells and delays renal cystic growth and progression in a $\mathrm{Pkd} 1$ heterozygous mouse model, suggesting that the inhibition of this way may also contribute to amelioration of renal function [42].

\section{Other Therapeutic Attempts}

Significant findings have been obtained in preclinical trials by using triptolide, an active diterpene used in Chinese medicine. Triptolide induces intracellular calcium release by a PC2-dependent mechanism and inhibit cell proliferation by restoring calcium signaling in mouse ADPKD cells [43]. Furthermore, triptolide improves renal function inhibiting early phases of cyst development in a mouse model of ADPKD [44].

Retrospective studies to evaluate the renoprotective effect of calcium channel blockers (CCB) and/or reninangiotensin-aldosterone system inhibitor (RAAS-I) by monitoring glomerular filtration rate (GFR) in ADPKD 
patients were also performed. No changes in GFR rate after treatment of ADPKD patients with RAAS-I compared with untreated were observed, but the administration of CCB causes an unfavorable effect on renal function with respect to $\mathrm{ADPKD}$ patients not receiving $\mathrm{CCB}$ treatment [45].

Recently, it was also reported that serum HDL-cholesterol may affect ADPKD progression. Thus, the management of cholesterol should be carefully considered in ADPKD patients [46].

In ADPKD patients the antidiuretic hormone, arginine vasopressin (AVP) stimulates cAMP accumulation contributing to cyst formation. Therefore, water intake should decrease cAMP levels by reduction of AVP activity. Consistently, acute water loading in ADPKD patients decreased urine cAMP concentration, but no significant decreasing in urine cAMP excretion after chronic water loading was observed [47]. Further studies will be needed to test the efficacy of water therapy.

\section{Future Potential Therapies}

Could a Multitarget Therapy Become More Successful in the Treatment of ADPKD?

Despite the positive results in preclinical models, findings obtained at the end of clinical trials by using sirolimus, everolimus, and related molecules currently in progress are not completely satisfactory. The mild results of sirolimus in human ADPKD patients can also be due to the administration of this compound at doses that are much too low. In fact, long-term treatment with sirolimus at low doses in ADPKD mouse models is not sufficient to inhibit mTOR activity in renal cystic tissue [48]. However, in $\mathrm{Pkd} 1^{\mathrm{nl}}$,nl ADPKD mice which express reduced levels of Pkd1 gene, the treatment with high- and low-dose sirolimus in the early disease stage significantly accelerated cyst regression [48]. A high dose of everolimus in clinical trials significantly reduces TKV, but it also induces side effects and does not improve renal function [8]. Therefore, further experiments researching new roads for the cure of ADPKD are required. In this regard, a combined treatment with different compounds blocking simultaneously more signaling pathways may be necessary, which should be more effective in restoring normal kidney parameters and minimizing side effects [48, 49]. This new approach should be addressed to the inhibition of cAMP, mTOR and EGFR pathways strongly involved in ADPKD development. Multitarget therapeutic approaches for the treatment of different pathologies es- pecially for cancer are emerging. In fact, combined inhibition of mTOR and ERK or mTOR and EGFR pathways shows synergistic effects and better efficacy than singletarget therapy in preclinical models for cancer and other diseases [50-52]. This goal could be, for instance, obtained by the combined use of sirolimus/everolimus, tolvaptan and EKI-785 that individually showed good results in ADPKD preclinical models. To evaluate possible synergistic effects, the procedure for multidrug treatment in preclinical models of ADPKD should provide for simultaneous or sequential administration of compounds, taking into account that the combined administration may have antagonizing or toxic effects [Aguiari, unpubl. data]. Compounds could be administrated daily or by pulse modality, and administration timing should be carefully evaluated in order to minimize side effects. It is also possibly necessary to try different combinations of targeted inhibitors for each selected pathway to test the best targeted drugs in terms of higher efficacy and lower toxicity. The treatment of ADPKD animals should be programmed in the early stage of disease, when the renal function is not yet compromised.

\section{Conclusions}

In this review, we propose alternative roads to treat ADPKD mainly addressed to the inhibition of cAMP, mTOR and EGFR pathways that are strongly involved in increased cell proliferation, fluid secretion and renal cyst development. Recently, multitargeted therapies for the treatment of different pathologies are emerging, especially when conventional single-target therapy failed. Therefore, for the future, multidrug approaches may provide new opportunities for the treatment of ADPKD.

\section{Acknowledgements}

Supported by CaRiCe, Italian MIUR COFIN 2008 and Regione Emilia Romagna (Ricerca Regione-Università) 2007-2009.

\section{Disclosure Statement}

The authors have no conflicts of interest to disclose. 


\section{References}

1 Gabow PA: Autosomal dominant polycystic kidney disease. N Engl J Med 1993;22:511-512.

$\checkmark 2$ Zheleznova NN, Wilson PD, Staruschenko A: Epidermal growth factor-mediated proliferation and sodium transport in normal and PKD epithelial cells. Biochim Biophys Acta 2011;1812:1301-1313.

$\checkmark 3$ Belibi FA, et al: Cyclic AMP promotes growth and secretion in human polycystic kidney epithelial cells. Kidney Int 2004;66:964-973.

$>4$ Shillingford JM, et al: The mTOR pathway is regulated by polycystin-1, and its inhibition reverses renal cystogenesis in polycystic kidney disease. Proc Natl Acad Sci USA 2006; 103:5466-5471.

5 Torres VE, et al, the TEMPO 3:4 Trial Investigators: Tolvaptan in patients with autosomal dominant polycystic kidney disease. $\mathrm{N}$ Engl J Med 2012 (E-pub ahead of print).

$\checkmark 6$ Hogan MC, et al: Randomized clinical trial of long-acting somatostatin for autosomal dominant polycystic kidney and liver disease. J Am Soc Nephrol 2010;21:1052-1061.

$>7$ Serra AL, et al: Sirolimus and kidney growth in autosomal dominant polycystic kidney disease. N Engl J Med 2010;363:820-829.

$>8$ Walz G, et al: Everolimus in patients with autosomal dominant polycystic kidney disease. N Engl J Med 2010;363:830-840.

$>9$ Hanaoka K, et al: Co-assembly of polycystin-1 and -2 produces unique cation-permeable currents. Nature 2000;408:990-994.

10 Nauli SM, et al: Polycystins 1 and 2 mediate mechanosensation in the primary cilium of kidney cells. Nat Genet 2003;33:129-137.

$\checkmark 11$ Praetorius HA, Spring KR: Bending the MDCK cell primary cilium increases intracellular calcium. J Membr Biol 2001;184:71-79.

12 Choi YH, et al: Polycystin-2 and phosphodiesterase $4 \mathrm{C}$ are components of a ciliary A-kinase anchoring protein complex that is disrupted in cystic kidney diseases. Proc Natl Acad Sci USA 2011;108:10679-10684.

13 Aguiari G, et al: Novel role for polycystin-1 in modulating cell proliferation through calcium oscillations in kidney cells. Cell Prolif 2008;41:554-573.

14 Yamaguchi T, et al: Cyclic AMP activates BRaf and ERK in cyst epithelial cells from autosomal-dominant polycystic kidneys. Kidney Int 2003;63:1983-1994.

$>15$ Terryn S, Ho A, Beauwens R, Devuyst O: Fluid transport and cystogenesis in autosomal dominant polycystic kidney disease. Biochim Biophys Acta 2011;1812:1314-1321.

16 Brook-Carter PT, et al: Deletion of the TSC2 and $\mathrm{PKD} 1$ genes associated with severe infantile polycystic kidney disease - a contiguous gene syndrome. Nat Genet 1994;8:328-332.

$\checkmark 17$ Dere R, Wilson PD, Sandford RN, Walker CL: Carboxy terminal tail of polycystin-1 regulates localization of TSC2 to repress mTOR. PLoS One 2010;5:e9239.
18 Weimbs T: Regulation of mTOR by polycystin-1: is polycystic kidney disease a case of futile repair? Cell Cycle 2006;5:2425-2429.

19 Distefano G, et al: Polycystin-1 regulates extracellular signal-regulated kinase-dependent phosphorylation of tuberin to control cell size through mTOR and its downstream effectors S6K and 4EBP1. Mol Cell Biol 2009;29:23592371.

20 Wilson PD: A plethora of epidermal growth factor-like proteins in polycystic kidneys. Kidney Int 2004;65:2441-2442.

21 Lowden DA, Lindemann GW, Merlino G, Barash BD, Calvet JP, Gattone VH: Renal cysts in transgenic mice expressing transforming growth factor-a. J Lab Clin Med 1994;124:386-394.

22 Aguiari G, et al: Polycystin-1 regulates amphiregulin expression through $\mathrm{CREB}$ and AP1 signalling: implications in ADPKD cell proliferation. J Mol Med 2012;90:1267-1282.

23 Du J, Wilson PD: Abnormal polarization of EGF receptors and autocrine stimulation of cyst epithelial growth in human ADPKD. Am J Physiol 1995;269:C487-C495.

24 Wilson SJ, et al: Inhibition of HER-2 (neu/ ErbB2) restores normal function and structure to polycystic kidney disease epithelia. Biochim Biophys Acta 2006;1762:647-655.

25 Torres VE, Wang X, Qian Q, Somlo S, Harris PC, Gattone VH: Effective treatment of an orthologous model of autosomal dominant polycystic kidney disease. Nat Med 2004;10: 363-364.

26 Reif GA, Yamaguchi T, Nivens E, Fujiki H, Pinto CS, Wallace DP: Tolvaptan inhibits ERK-dependent cell proliferation, $\mathrm{Cl}^{-}$secretion, and in vitro cyst growth of human ADPKD cells stimulated by vasopressin. Am J Physiol Renal Physiol 2011;301:F1005F1013.

27 Masyuk TV, Masyuk AI, Torres VE, Harris PC, Larusso NF: Octreotide inhibits hepatic cystogenesis in a rodent model of polycystic liver disease by reducing cholangiocyte adenosine 3',5'-cyclic monophosphate. Gastroenterology 2007;132:1104-1116.

28 Hogan MC, et al: Somatostatin analog therapy for severe polycystic liver disease: results after 2 years. Nephrol Dial Transplant 2012; 27:3532-3539.

29 Caroli A, et al: effects of somatostatin analogue octreotide. Clin J Am Soc Nephrol 2010; 5:783-789.

30 Aguiari G, et al: Deficiency of polycystic kidney disease-1 gene (PKD1) expression increases $\mathrm{A}_{3}$ adenosine receptors in human renal cells: implications for cAMP-dependent signalling and proliferation of PKD1-mutated cystic cells. Biochim Biophys Acta 2009;1792: 531-540.
31 Yamaguchi T, Reif GA, Calvet JP, Wallace DP: Sorafenib inhibits cAMP-dependent ERK activation, cell proliferation, and in vitro cyst growth of human ADPKD cyst epithelial cells. Am J Physiol Renal Physiol 2010; 299:F944-F951.

32 Yamaguchi T, et al: cAMP stimulates the in vitro proliferation of renal cyst epithelial cells by activating the extracellular signal-regulated kinase pathway. Kidney Int 2000;57:14601471.

33 Shibazaki S, et al: Cyst formation and activation of the extracellular regulated kinase pathway after kidney-specific inactivation of Pkd1. Hum Mol Genet 2008;17:1505-1516.

34 Omori S, et al: Extracellular signal-regulated kinase inhibition slows disease progression in mice with polycystic kidney disease. J Am Soc Nephrol 2006;17:1604-1614.

35 Wahl PR, Serra AL, Le Hir M, Molle KD, Hall MN, Wüthrich RP: Inhibition of mTOR with sirolimus slows disease progression in Han:SPRD rats with autosomal dominant polycystic kidney disease (ADPKD). Nephrol Dial Transplant 2006;21:598-604.

>36 Wu M, Wahl PR, Le Hir M, Wackerle-Men Y, Wuthrich RP, Serra AL: Everolimus retards cyst growth and preserves kidney function in a rodent model for polycystic kidney disease. Kidney Blood Press Res 2007;30:253-259.

37 Perico N, et al: Sirolimus therapy to halt the progression of ADPKD. J Am Soc Nephrol 2010;21:1031-1040.

- 38 Grantham JJ, Bennett WM, Perrone RD: mTOR inhibitors and autosomal dominant polycystic kidney disease. N Engl J Med 2011; 364:286-289.

39 Torres VE, et al: EGF receptor tyrosine kinase inhibition attenuates the development of PKD in Han:SPRD rats. Kidney Int 2003;64: 1573-1579.

40 Gao J, et al: Curcumin inhibits renal cyst formation and enlargement in vitro by regulating intracellular signaling pathways. Eur J Pharmacol 2011;654:92-99.

-41 Leonhard WN, et al: Curcumin inhibits cystogenesis by simultaneous interference of multiple signaling pathways: in vivo evidence from a Pkd1-deletion model. Am J Physiol Renal Physiol 2011;300:F1193-F1202.

-42 Elliott J, Zheleznova NN, Wilson PD: c-Src inactivation reduces renal epithelial cell-matrix adhesion, proliferation, and cyst formation. Am J Physiol Cell Physiol 2011; 301:C522-C529.

43 Leuenroth SJ, et al: Triptolide is a traditional Chinese medicine-derived inhibitor of polycystic kidney disease. Proc Natl Acad Sci USA 2007;104:4389-4394.

44 Leuenroth SJ, et al: Triptolide reduces cystogenesis in a model of ADPKD. J Am Soc Nephrol 2008;19:1659-1662. 
45 Mitobe M, et al: Clinical effects of calcium channel blockers and renin-angiotensin-aldosterone system inhibitors on changes in the estimated glomerular filtration rate in patients with polycystic kidney disease. Clin Exp Nephrol 2010;14:573-577.

46 Torres VE, et al: Potentially modifiable factors affecting the progression of autosomal dominant polycystic kidney disease. Clin J Am Soc Nephrol 2011;6:640-647.
47 Barash I, Ponda MP, Goldfarb DS, Skolnik EY: A pilot clinical study to evaluate changes in urine osmolality and urine cAMP in response to acute and chronic water loading in autosomal dominant polycystic kidney disease. Clin J Am Soc Nephrol 2010;5:693-697.

48 Novalic Z, et al: Dose-dependent effects of sirolimus on mTOR signaling and polycystic kidney disease. J Am Soc Nephrol 2012;23: 842-853.

49 Chang MY, Ong AC: Mechanism-based therapeutics for autosomal dominant polycystic kidney disease: recent progress and future prospects. Nephron Clin Pract 2012;120:c25c34.

50 Schmid K, et al: Dual inhibition of EGFR and mTOR pathways in small cell lung cancer. $\mathrm{Br}$ J Cancer 2010;103:622-628.
51 Jin N, Jiang T, Rosen DM, Nelkin BD, Ball DW: Synergistic action of a RAF inhibitor and a dual PI3K/mTOR inhibitor in thyroid cancer. Clin Cancer Res 2011;17:6482-6489.

52 Lin JT, et al: Differential mTOR and ERK pathway utilization by effector CD4 T cells suggests combinatorial drug therapy of arthritis. Clin Immunol 2012;142:127-138.

53 Marcotte R, Zhou L, Kim H, Roskelly CD, Muller WJ: c-Src associates with ErbB2 through an interaction between catalytic domains and confers enhanced transforming potential. Mol Cell Biol 2009;29:5858-5871. 